\title{
Campagne de mesures au moyen de traceurs radioactivables et fluorescents sur la canal Jamrao (Sindh, Pakistan)
}

\author{
Alexandre Vabre \\ Doctorant, CEA Centre de Saclay, DTA/DAMRI/SAR
}

\section{Résumé}

Cet article présente une campagne de mesures au moyen de techniques de traceurs. On appelle traceur un produit facilement identifiable qui, introduit dans une population de phases à tracer, a le même comportement chimique ou hydrodynamique que l'une d'entre elles.

Sur le système d'irrigation sélectionné (Jamrao), une claire tendance au dépôt solide a été identifiée pendant le fonctionnement passé. Forte de ces observations, une campagne de mesures par traceurs a été spécialement élaborée. Le choix d'utiliser des traceurs a été motivé par la sensibilité de la technique, en effet les phénomènes de dépôt solide sont très lents. Dans la plupart des cas les durées d'observation doivent être de l'ordre d'années ou même de décennies pour obtenir des évolutions significatives. Alors qu'en utilisant des traceurs, même de très petits dépôts de matière peuvent être qualifiés et surtout quantifiés.

Dans le but d'expliquer les dépôts observés lors de la campagne de mesures, une composante topographique et hydraulique est ajoutée. En effet, les phénomènes de transport solide sont expliqués en grande part par le comportement de l'écoulement liquide. Dans la dernière partie de l'article, une approche de modélisation globale des phénomènes de dépôt développée pour les besoins du travail est élaborée sur les résultats de la campagne de mesures.

\begin{abstract}
This paper presents a measurement campaign using tracer techniques. A tracer is a material easy to identify which, once introduced in a population of physical phases to trace, has the same chemical or hydrodynamical behavior than one of them.

On the selected irrigation system (Jamrao), a clear tendency to the sediment deposition has been identified during the past functioning. Based on these observations, a measurement campaign using tracers has been elaborated. This technique has been chosen because of its sensitivity, indeed the sediment deposition phenomena are very slow ones. In most of the cases the observation durations have to be in the magnitude of years or even decades to obtain significant evolutions. Whereas using tracer techniques, even very small depositions of materials can be qualified and especially quantified.

In order to explain the observed deposition during the measurement campaign, a topographic and hydraulic component is added. Indeed, the sediment transport phenomena are mainly explained by the liquid flow behavior. In the last part of the paper, a global modeling approach of the deposition phenomena, which has been developed for the needs of the work, is elaborated on the measurement campaign results.
\end{abstract}




\section{Introduction}

Cette campagne de mesures est incluse dans un programme de recherche en sédimentologie dynamique sur les systèmes de canaux d'irrigation, avec une application particulière au Pakistan. C'est un projet en commun entre l'IMFT', le CEA $^{2}$, l'IIMI-Pakistan ${ }^{3}$, le Cemagref ${ }^{4}$ et l'ISRIP $^{5}$ qui a été initié en 1995. Depuis 1997, il est le siège de deux thèses de recherche. Ce projet vise à étudier les phénomènes sédimentaires dans des systèmes de canaux d'irrigation au moyen de deux approches distinctes et complémentaires. Une première dite mécaniste repose sur une explication déterministe des phénomènes sédimentaires sur des biefs linéaires du système d'irrigation. Une seconde dite globale repose sur une description et une explication globale des phénomènes sédimentaires dans l'ensemble des parties constitutives du système d'irrigation. Les deux approches travaillent à différentes échelles temporelles et spatiales pour les phénomènes sédimentaires. En effet, typiquement les pas de temps et d'espace de l'approche de modélisation mécaniste sont respectivement de 7 jours et $100 \mathrm{~m}$ tandis qu'ils sont de 10 ans et $10 \mathrm{~km}$ pour l'approche globale.

La présente campagne de mesures fait partie intégrante de l'approche globale, les objectifs et méthodologie détaillés de la campagne font l'objet d'une note technique (Vabre, 1998).

Deux méthodes sont successivement employées afin de représenter et expliquer globalement les phénomènes sédimentaires dans le système d'irrigation:

$\checkmark$ Suivi topographique, sur de longues périodes de temps de plusieurs décennies, pour observer des variations significatives des paramètres topographiques. II va de soi que la mesure des paramètres hydrauliques et sedimentologiques pendant le suivi topographique est nécessaire pour son explication.

$\checkmark$ Utilisation de techniques de traceurs, pendant des périodes de temps courtes de quelques mois. Il va de soi que la mesure des paramètres topographiques et hydrauliques pendant le transit des traceurs est nécessaire pour son explication.

La première méthode est basée sur l'analyse des données existantes du site d'étude et concerne la période de fonctionnement comprise entre 1932 et 1982. Cela représente donc un observation des paramètres topographiques pendant une durée de 50 ans. La seconde méthode est quant à elle mise en œuvre sur les seules données traceurs disponibles de la présente campagne 1998 sur une durée de 4 mois. Les paramètres hydrauliques correspondants à ces deux périodes sont pris comme la moyenne des observations.

\footnotetext{
'Institut de Mécanique des Fluides de Toulouse, France

${ }^{2} \mathrm{CEA}$ :Commissariat à l'Energie Atomique, France

${ }^{3}$ International Water Management Institute, Pakistan

${ }^{4}$ La recherche pour l'ingénierie de l'agriculture et de l'environnement, France

${ }^{5}$ International Sedimentation Research Institute of Pakistan, Pakistan
} 


\section{Protocole expérimental}

\subsection{Site d'étude}

Un cas d'étude de système de canaux d'irrigation (Jamrao) a été sélectionné dans la province du Sindh au Pakistan. Une collecte de données sur le fonctionnement passé du système a permis d'identifier une tendance claire au dépôt solide. Dans les biefs amont, la hauteur du dépôt de sables a été de plus $3,0 \mathrm{~m}$ pour un tirant d'eau de conception de l'ordre de $2,6 \mathrm{~m}$. Une présentation de la gamme de variation des différents paramètres dans le système est proposée dans le tableau 1.

\begin{tabular}{llll}
\hline paramètre & définition & valeur minimale & valeur maximale \\
\hline$Q\left(\mathrm{~m}^{3} / \mathrm{s}\right)$ & débit & 6,60 & 112,67 \\
$V(\mathrm{~m} / \mathrm{s})$ & vitesse & 0,42 & 1,22 \\
$R_{H}(\mathrm{~m})$ & rayon & 1,16 & 2,74 \\
& hydraulique & & \\
$J(\mathrm{~m} / \mathrm{m})$ & pente d'énergie & $8,0010^{-5}$ & $1,9610^{-4}$ \\
$d_{s 0}(\mu \mathrm{m})$ & diamètre médian & 80 & 188 \\
\hline \multicolumn{4}{c}{ tableau 1 : gamme de variation des paramètres dans le système } \\
& (range of variation of the parameters in the system)
\end{tabular}

\subsection{Détermination des points de mesure}

Le système d'irrigation retenu pour l'étude (Jamrao) est composé de différents types de canaux d'amont en aval du système. Seuls les canaux amonts dans le système font l'objet de l'étude qui suit. Du fait de leurs longueurs très importantes, des critères d'identification de zones homogènes ont été développés et sont au nombre de 3 . Le premier critère est purement hydraulique tandis que les deux autres sont sédimentologiques. Ce premier critère sert à garantir des conditions stables d'écoulement liquide dans la zone identifiée (Yang, 1972). Le deuxième critère impose au bief d'avoir une longueur suffisante pour permettre le dépôt d'une particule y entrant (Hazen, 1904). Et le troisième critère impose à la longueur du bief de ne pas excéder $95 \%$ de la longueur concernée par le dépôt, celle-ci étant définie à partir d'une loi de transport solide hors équilibre (Daubert \& Lebreton, 1967).

Les emplacements finaux des points de mesures sont alors déterminés pour représenter un dépôt moyen dans le bief homogène concerné. Cela implique de localiser le point à $L_{m o y}=15 \% . L_{\max }$ à partir de la tête du bief. Chaque point de mesure est représenté par une surface élémentaire d'investigation, la mesure en ce point est ensuite extrapolée au bief homogène entier.

Les critères de découpage précédents ont été appliqués sur le jeu de données de dépôt par variations topographiques dans le site d'étude retenu et ceci a permis d'identifier 21 zones (biefs) homogènes et 21 points de mesures associés aux canaux de grande dimension. A cela s'ajoute un nombre de 13 points de mesures sur des canaux de petite dimension. 


\subsection{Particules traçantes des sables}

Forte des observations de la dynamique de dépôt solide global du système de canaux d'irrigation choisi (Jamrao), une campagne de mesures au moyen de techniques de traceurs est développée pour observer et expliquer ces phénomènes. De façon pratique, les traceurs sont représentatifs des matières solides qui se déposent dans le système d'irrigation, et la campagne de mesures vise à montrer la distribution spatiale du dépôt de traceurs. Les traceurs sont injectés en tête du système. Puis, pendant une durée donnée, la quantité de traceur déposée est mesurée en des stations déterminées dans le système.

Afin de mener le travail d'explication des masses de traceurs déposées, des mesures topographiques, hydrauliques et sédimentologiques sont nécessaires pendant la campagne de mesures. Une équipe de MISRIP a été employée pour ce travail. Cette équipe a également aidé au prélèvement des matériaux déposés (contenant les traceurs) dans le système.

Les particules traçantes sont choisies de deux natures et leur répartition granulométrique est ajustée à celle des particules naturelles dủ système. En premier lieu, des traceurs radioactivables sont utilisés, ce sont des matériaux inertes (non radioactifs), en général des verres contenant de façon massique et irréversible un élément dopant (facilement radioactivable) dans sa forme isotopique stable. Cette technique est en cours de développement au CEA et a été testée pendant la campagne de mesures. Dans notre cas, le site d'étude étant naturel et en vraie grandeur, le traceur est amené à être très fortement dilué. $\mathrm{Du}$ fait des éléments présents de façon naturelle dans le site d'étude, l'élément dopant choisi est l'iridium. C'est de toute façon un élément présent en très faible teneur dans l'environnement (Alvarez et al., 1980) et qui présente des caractéristiques particulièrement intéressantes pour le dosage en analyse par activation (Huet, 1998a). En second lieu, des traceurs fluorescents sont utilisés, ce sont des sables naturels peints avec une peinture rouge dont la fluorescence est excitée par éclairage ultraviolet.

Ces particules traçantes sont alors injectées de façon ponctuelle (Tola, 1982 et Brisset, 1994) en tête du système et prélevées dans des échantillons de sable naturel aux points de mesure du système. Les traceurs sont ensuite dosés dans ces échantillons naturels.

\subsection{Mesures terrain}

Les travaux de mesures qui ont été conduits consistent à mesurer ou collecter :

$\checkmark$ la section en travers et le niveau d'eau $: R_{H}$ (rayon hydraulique)

$\checkmark$ la pente de la ligne d'eau : $J$ (gradient d'énergie)

$\checkmark$ la vitesse moyenne d'écoulement et débit de liquide : $V$ et $Q$ (vitesse et débit)

$\checkmark$ des échantillons de sédiments en suspension : $C$ (concentration)

$\checkmark$ des échantillons de sédiments de lit du canal : $d_{s 0}$ (diamètre médian)

La durée du programme terrain a été de 12 semaines, avec une observation complète du site en 2 semaines, soit 6 jeux de données. 


\subsection{Analyse des échantillons solides prélevés}

\subsubsection{Granulométrie}

Les échantillons de sédiments de lit du canal, après collecte ont été séchés et une mesure de la répartition granulométrique a été conduite dans les laboratoires de l'ISRIP. Le nombre total d'échantillons du lit du canal collectés sur le terrain a été de 204 (34 stations par 6 prélèvements).

\subsubsection{Traceurs fluorescents}

Une instrumentation spécifique par imagerie a été développée pour la mesure des traceurs fluorescents. L'idée principale est de rendre quantitative cette méthode qui est dans la plupart des cas purement qualitative. Cette instrumentation a été développée dans le cadre d'un stage de DEA (Doublier \& Vabre, 1999) et les analyses ont été réalisées dans les locaux du CEA à Saclay.

La méthode de mesure consiste à effectuer une cartographie d'un échantillon de l'ordre de $20 \mathrm{~g}$ au moyen d'une caméra vidéo haute résolution. Puis de localiser les grains fluorescents et de mesurer leur taille, dans notre cas, une résolution de $14 \mu \mathrm{m}$ au pixel avec des images de 739 par 575 pixels ont été utilisées. Cela implique de collecter 2592 images par échantillon.

\subsubsection{Traceuis radioactivables}

La méthode de mesure de tels traceurs est l'analyse par activation neutronique. Elle consiste à activer l'élément dopant traceur sous flux neutronique. Cet élément, rendu radioactif, est ensuite mesuré par spectrométrie gamma.

Le choix technologique pour l'irradiation a été d'utiliser un réacteur nucléaire dans lequel le flux en neutrons thermiques, les plus efficaces pour l'activation, est le plus fort. En effet l'irradiation avec des sources de neutrons n'était pas envisageable pour la présente manipulation du fait du taux de dilution en traceurs très important et des flux limités de telles sources (Huet, 1998b). Une durée d'irradiation d'une heure est fixée comme un maximum avec une masse d'échantillon de l'ordre de $50 \mathrm{~g}$ du fait des différents éléments présents de façon naturelle dans l'échantillon et qui peuvent le rendre très actif et donc difficile à manipuler. De part cette contrainte, il est également nécessaire de laisser décroître l'échantillon analysé pour rendre la mesure en spectrométrie gamma faisable.

Toutes les analyses des échantillons ont été réalisées dans un centre nucléaire pakistanais du fait d'une plus grande facilité pour acheminer les échantillons et de la volonté de développer une collaboration avec le centre au Pakistan. 


\section{Résultats de mesures}

\subsection{Mesures topographiques et hydrauliques}

Le jeu de données topographiques et hydrauliques est synthétisé par la valeur de la capacité de transport solide $\eta$ (voir l'équation 1). On observe une large gamme pour les valeurs de $\eta$. Cela est dû au fait que le système d'irrigation choisi pour le travail est observé dans sa presque totalité, offrant ainsi une gamme très large de régime d'écoulement hydraulique. Ce point est intéressant car cela doit permettre d'observer également une large gamme tendance de dépôt.

Il est observé une différence relative modérée de l'ordre de $15 \%$ entre la valeur moyenne de $\eta$ et la valeur de début de campagne ayant provoqué le dépôt ce qui vient conforter l'hypothèse quant au choix de représenter les conditions hydrauliques d'écoulement par leurs valeurs moyennes.

\subsection{Mesures traceiurs}

Les analyses par activation neutronique ont été conduites au Pakistan. Du fait de fortes erreurs sur les résultats de ces analyses, deux mesures correctives ont été prises. D'une part, une technique de dosage des traceurs fluorescents aussi précise que possible a été développée (voir $\S 2.5 .2$ ), et d'autre part une technique de correction des résultats au moyen d'échantillons étalons est proposée. L'analyse par activation a été conduite sur 300 échantillons, répartis en 204 échantillons prélevés sur le terrain, 46 doublons de ces échantillons et 50 étalons. Sur les doublons des échantillons prélevés, une erreur relative de $40 \%$ en moyenne est observée.

La correction des résultats de l'analyse par activation repose sur un jeu d'échantillons étalons dans lesquels une quantité connue de traceur activable a été introduite. Le protocole de mesure suivi pour l'analyse de ces échantillons est le même que celui des échantillons prélevés lors de la campagne. Sur les échantillons étalons, des calculs de moyenne et variance ont été conduits. Des valeurs aberrantes ont ensuite été éliminées pour minimiser la variance (Neuilly, 1993 et AFNOR, 1999). On peut observer que la réponse en masse mesurée n'est jamais nulle et cela même pour une masse réelle nulle en traceur activable, la réponse minimum est de l'ordre de $70 \mathrm{ng}$. De plus, les résultats mesurés surestiment toujours la masse réelle d'un facteur $18 \mathrm{ng} / \mathrm{ng}$. Les résultats mesurés ont donc été corrigés en tenant compte de ces observations. Le choix de corriger les résultats des analyses a été motivé par le caractère pragmatique de l'étude. En plus de la correction présentée ci-dessus sur les analyses des traceurs activables, un certain nombre de valeurs aberrantes ont dû être éliminée en respectant le principe de minimisation de la variance présenté précédemment. Le jeu de résultats ainsi obtenu est présenté sur les figures qui suivent.

La mesure en teneur de traceurs fluorescents dans les échantillons a été conduite sur les 204 échantillons prélevés lors de la campagne de mesures. L'allure des teneurs en traceurs dans les échantillons est représentée sur la figure 1 et la figure 2 qui correspondent respectivement aux canaux de grande et petite 
dimensions. Sur les canaux de grande dimension, 21 points de mesures ont été identifiés au moyen des critères de découpage en éléments homogènes et sont nommés: Ji, Di, Si et Wi. Tandis que sur les canaux de petite dimension, 13 points de mesures ont été identifiés de façon arbitraire et sont nommés: SD, RA, BI, PA, MA, DO, BA, LA et SAi.

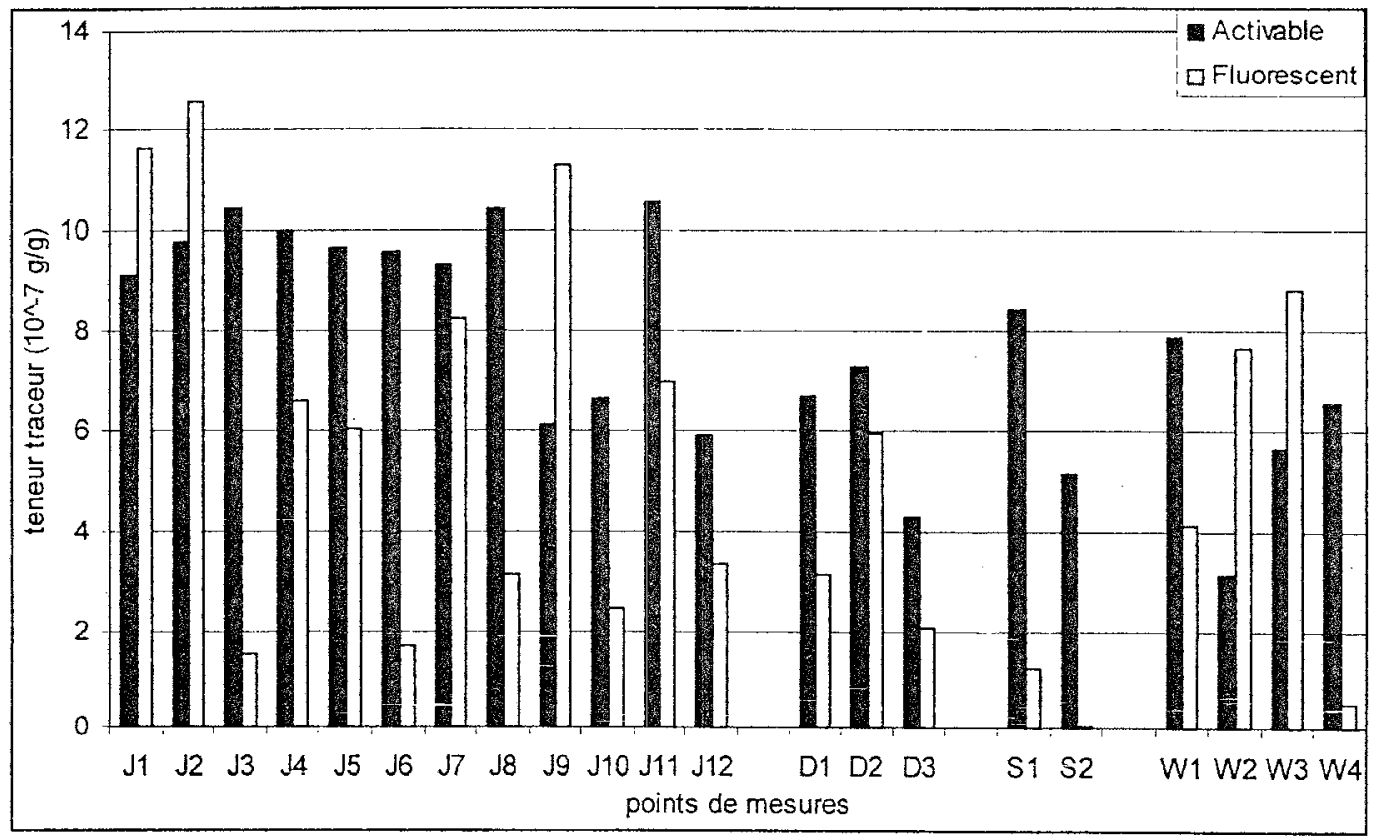

figure 1 : teneur en traceur dans les canaux de grande dimension (tracer content in the wide dimension canals)

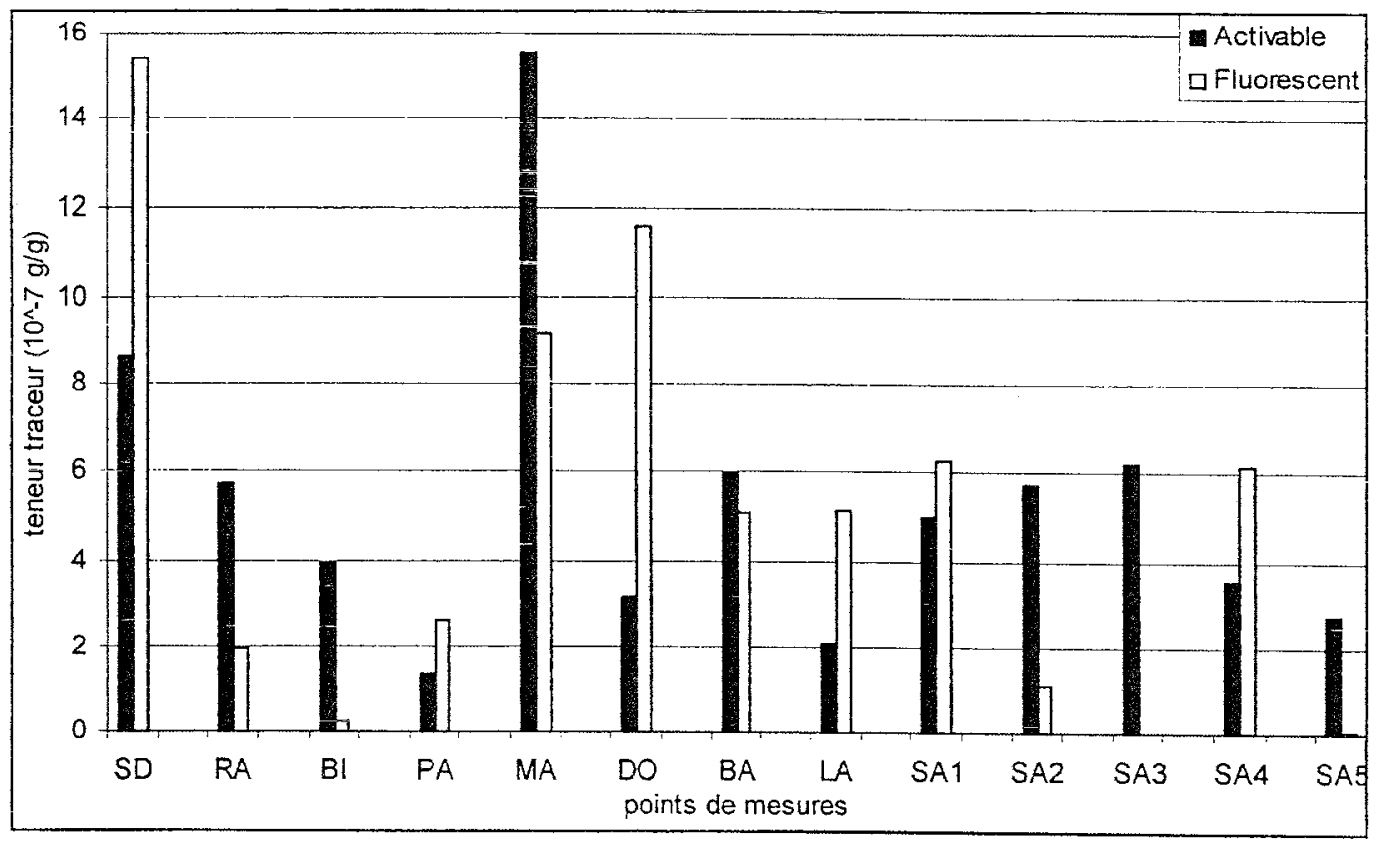

figure 2 : teneur en traceur dans les canaux de petite dimension (tracer content in the canals of the system) 
Dans les canaux de grande dimension, les résultats peuvent être assez comparables entre les deux méthodes. Cela s'explique en grande part par la granulométrie élevée des particules qui sont mesurées et donc de la réponse en masse de traceurs assez importante dans les échantillons analysés. Ce n'est pas le cas dans les canaux de petite dimension dans lesquels certaines valeurs mesurées peuvent sont très faibles, ou porter sur des granulométries assez fines, et donc induire des différences très importantes.

La comparaison des deux méthodes de mesure est globalement assez encourageante. En effet, les dynamiques globales de dépôt observées sont compatibles. Néanmoins de grandes différences peuvent être observées dans certains canaux du système et en particulier dans les canaux de petite dimension. Les résultats des traceurs activables sont préférés dans la plupart des cas car ils sont a priori plus fiables. En effet la technique de marquage en masse de ces traceurs a un caractère définitif. Ce n'est pas le cas pour le marquage de surface des traceurs fluorescents. Dans l'écoulement naturel des grains peuvent entrer en contact et éroder la peinture de surface. Ces phénomènes d'érosion du marquage ont pu être observés sur tous les échantillons réels situés loin spatialement du point d'injection (points aval) et prélevés longtemps après l'injection (en fin de campagne). En effet, dans ces cas, une réponse nulle en traceurs fluorescents est observée dans de nombreux cas tandis que les traceurs radioactivables indiquaient une réponse contradictoire. L'intérêt de ces traceurs fluorescents est tout de même de disposer d'informations complémentaires et de pouvoir valider les hypothèses quant aux éliminations des valeurs aberrantes de l'analyse des traceurs activables.

\section{Exploitation des résultats : modélisation numérique}

\subsection{Variables modélisées et loi}

Une approche de modélisation, dédiée aux traceurs est développée, elle est intitulée GSM (Global Sediment Model) et est basée sur les lois classiques du transport solide. Cette approche travaille sur des pas d'espace (quelques kilomètres) et de temps (quelques années) très importants pour pouvoir être directement appliquée par les gestionnaires d'irrigation.

Il s'ensuit une présentation sommaire des concepts de base de ce modèle. Tout d'abord, une capacité de transport de sédiments (Engelund \& Hansen, 1967) de l'écoulement liquide est définie, c'est la valeur à l'équilibre $q_{s}^{*}$ du débit solide exprimée par :

$$
q_{s}{ }^{*}=0,05 \cdot g^{-1 / 2} \cdot(s-1)^{-2} \cdot V^{2} \cdot R_{H}^{3 / 2} \cdot J^{3 / 2} \cdot d_{s 0}{ }^{-1}
$$

Puis, cette valeur est normée par le débit solide par unité de largeur de canal pour donner une variable $\eta$ (adimensionnelle), exprimée par :

$$
\eta=q_{s}^{*} / q_{2}
$$

Enfin, le taux de dépôt $D / A$ (dépôt $D$ sur apports $A$ de matières solides, adimensionnel) est exprimé comme une fonction de $\eta$. D'après des études antérieures (Vabre \& Le Guennec, 1999), des seuils sont observés sur les valeurs 
de $\eta$ pour différentes dynamiques de $D / A$, comme cela est présenté sur la figure 3 . Il est admis physiquement que, d'une part, $D / A(\eta=0)=1$, en effet, pour une capacité de transport nulle, un bief stocke tous ses apports en matières solides, et que d'autre part, il existe une valeur d'équilibre $\eta_{c}$ telle $D / A\left(\eta_{d}\right)=0$.

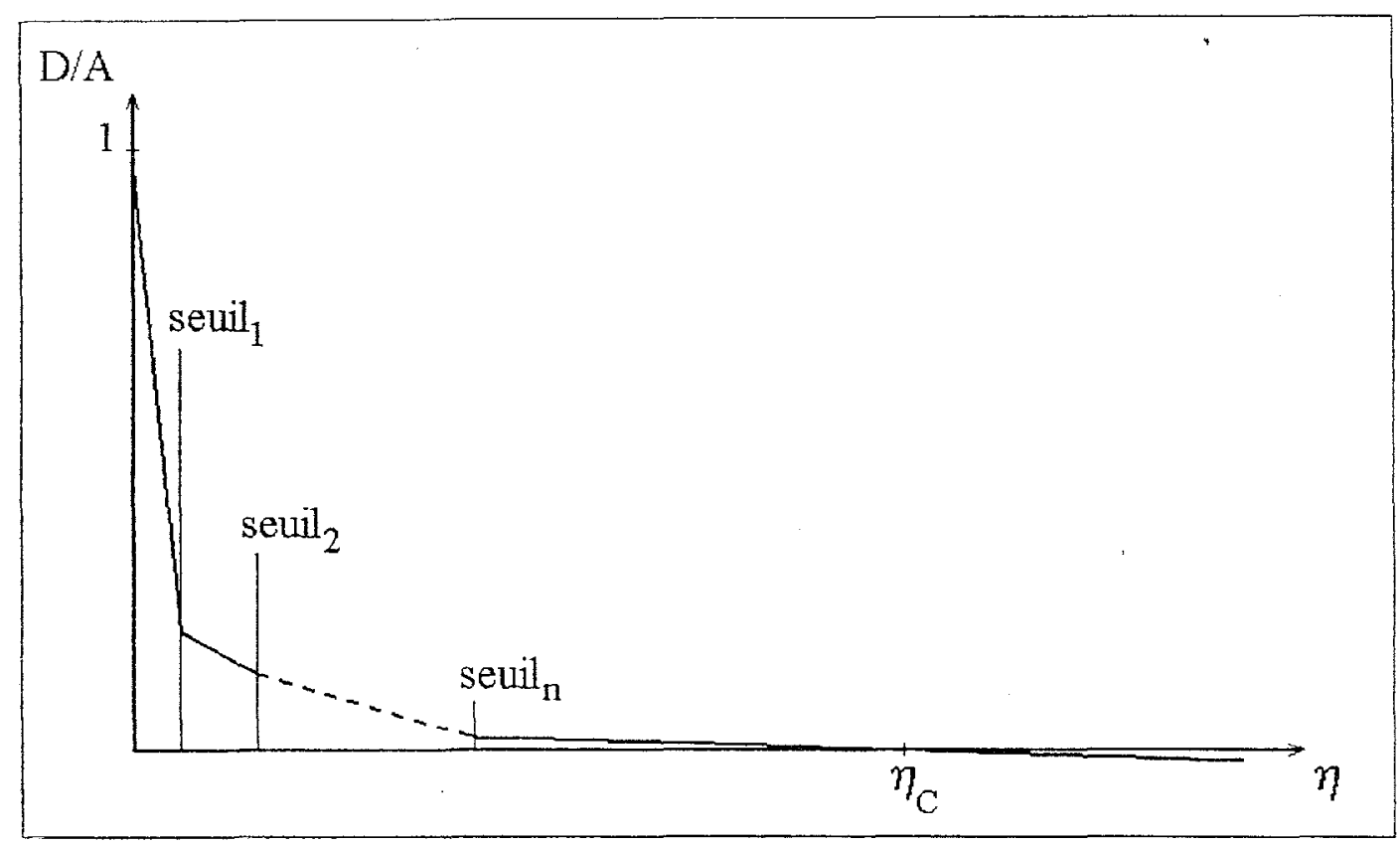

figure 3 : allure générique de $\mathrm{D} / \mathrm{A}$ en fonction de $\eta$

(generic shape of D/A versus $\eta$ )

\subsection{Résultat sur les données de la campagne de mesures}

Les résultats des teneurs en traceurs des différentes portions du système au point de prélèvement sont ramenés à un dépôt équivalent $D$ dans le bief concerné par une approximation de dépôt homogène. En effet, les prélèvements ne sont réalisés que sur des surfaces réduites de lit des canaux. Il convient donc de convertir le résultat local à résultat équivalent à toute la surface concernée du bief. Ces dépôts sont ensuite normés par les apports $A$ à chaque bief pour construire la fonction taux de piégeage $D / A$ de chaque bief homogène. Et ces valeurs sont tracées en fonction de la capacité de transport solide $\eta$ pour identifier les paramètres de la loi de dépôt $D / A(\eta)$, voir la figure 4 . 


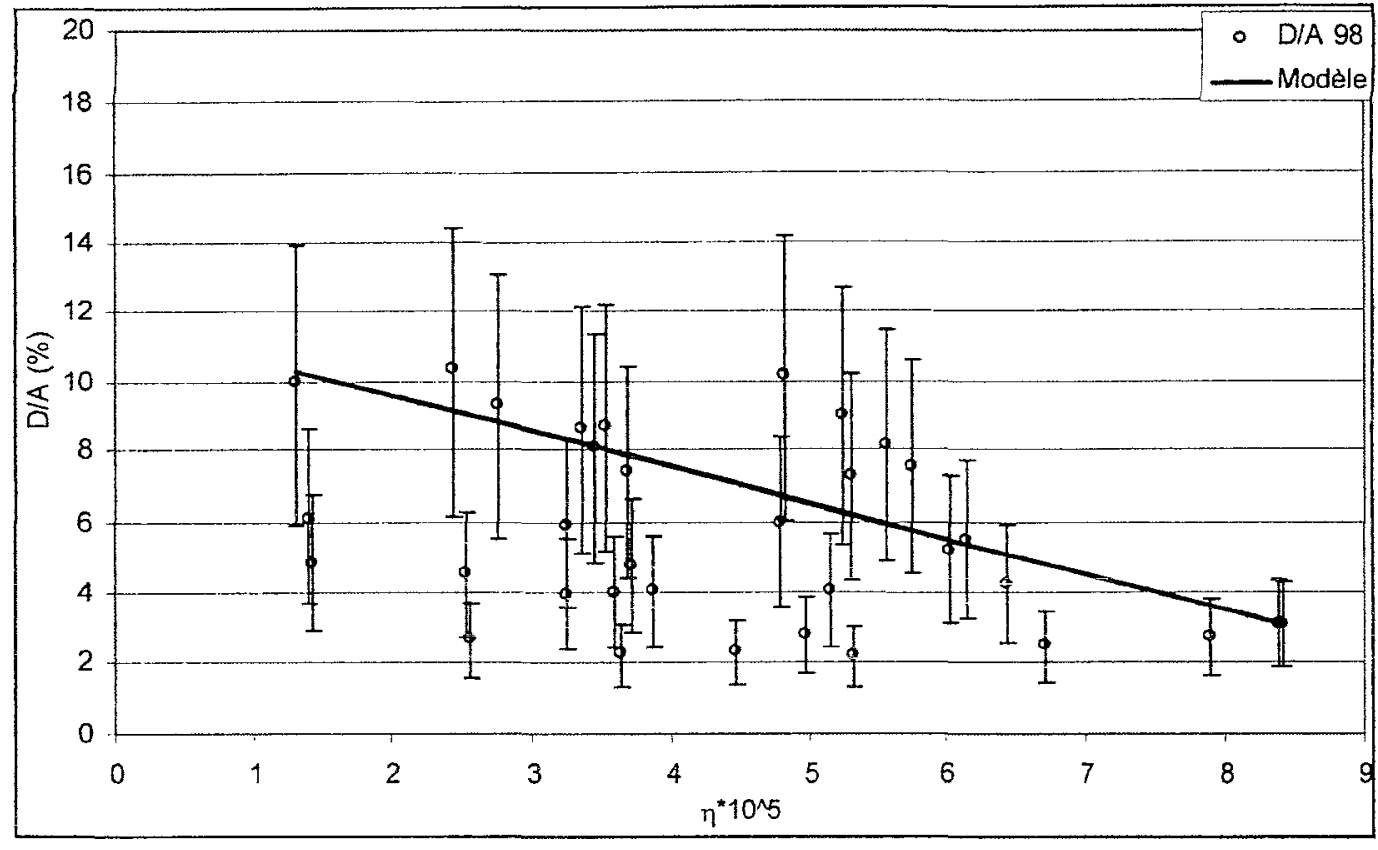

figure 4 : fonction piegeage $\mathrm{D} / \mathrm{A}$ fonction de la capacité de transport $\eta$ (trapping efficiency function $D / A$ versus transport capacity $\eta$ )

Les résultats sont très encourageants car l'allure de la courbe de dépôt obtenue correspond à l'allure générique de la figure 3 . Il n'est pas observé de seuil pour ces valeurs de $\eta$ asse $z$ importantes $\left(\eta>10^{-5}\right)$.

La masse totale équivalente de dépôt qui a été calculée dans les canaux du système par l'approximation de dépôt homogène représente la moitié de la masse injectée de traceur. Comme c'est également la moitié des canaux du système qui a été observée pendant le programme terrain, on peut considérer que toute la masse de traceur s'est déposée et que l'approximation de dépôt homogène est valide.

On peut tout de même observer des différences entre les valeurs mesurées et le lissage, en moyenne l'erreur relative est de $30 \%$. Mais l'approche de modélisation globale se contente de telles erreurs du fait du domaine d'étude et de ses applications. En effet, des erreurs de l'ordre de $50 \%$ sont courantes en transport solide. Et de plus, le but final de cet outil est d'être utilisé pour la gestion de systèmes d'irrigation. Sa mise ne œuvre n'est en général faite qu'en terme de tendance en comparant différents scénarios hypothétiques de gestion et en choisissant le meilleur d'entre eux sur un critère lié au transport solide, par exemple la masse déposée dans le système. 


\section{Conclusion}

Le jeu de données collecté dans le cadre de ce travail est minimal et suffisant, à la fois en durée de l'expérimentation et en résolution spatiale. Ce choix était volontaire et il semble que les résultats obtenus soient forts satisfaisants pour mettre en oeuvre une approche de modélisation GSM. Les erreurs obtenues entre le lissage d'une loi de dépôt et les mesures terrain par traceurs sont forts acceptables, et spécialement en vue des applications de gestion de l'irrigation. Il n'aurait pas été possible d'effectuer le même type de mesures sur le système de canaux d'irrigation choisi sans l'aide des techniques de traceur. En effet, pour obtenir des évolutions significatives sur le phénomène de dépôt, les observations de variations topographiques ont été réalisées pendant 50 ans tandis que la campagne traceurs n'a durée que 4 mois. La méthode de mesures par traceurs radioactivables est en cours de développement, l'expérimentation terrain présentée dans cet article est innovante. Les résultats sont très prometteurs et de nouvelles applications sont envisageables pour observer des phénomènes physiques similaires à ceux qui sont observés dans la présente étude, à savoir les dépôts.

La loi de dépôt calée sur les dépôts de traceurs de la présente campagne a été comparée à celle obtenue par des mesures du dépôt par variation topographique. Les lois obtenues sont très similaires, ce résultat vient à la fois conforter la loi obtenue par variations topographiques et souligner l'iñtérêt des mesures au moyen des techniques de traceurs. En effet, pour l'étude d'un système d'irrigation sur lequel aucune données ne sont disponibles, la mise en œuvre seule d'un campagne traceurs permet de rendre compte de son comportement en terme de dépôt au moyen d'un loi de dépôt.

\section{Références}

AFNOR (1999). Guide pour l'expression de l'incertitude de mestre. Norme Française NF ENV 13005. Indice de classement X 07-020, 105 p.

Alvarez, L.W., Alvarez, W., Asaro, F. \& Michel, H.V. (1980). Extraterrestrial cause for the cretaceous-tertiary extinction. Experimental results and theoretical interpretation. Journal Science, Volume 208, Number 4448, pp. 1095-1108.

Bagnold, R. (1966). An approach to the sediment transport from general physics. Geological Survey Professional Paper 422-1, United States Government Printing Office, Washington Edition, $37 \mathrm{p}$.

Brisset, P. (1994). L'application des radioéléments en sédimentologie. Note Technique CEA, DAMRI/SAR/S/NT/ 94-410, 28 p.

Daubert, A. \& Lebreton, J.C. (1967). Etude expérimentale et sur modèle mathématique de quelques aspects des processus d'érosion des lits alluvionnaires en régime permanent et non permanent. Twelfth Congress of the International 
Association for Hydraulic Research. Affouillements locaux du lit à l'aval des ouvrages hydrauliques, Vol. 3, pp. 26-37.

Doublier, A. \& Vabre, A. (1999). Mesure de la teneur en traceur fluorescent dans des échantillons de sable pour des applications sédimentaires. Note Technique CEA, DAMRI/SAR/S/NT/99-413, $60 \mathrm{p}$.

Engelund, F.A. \& Hansen, E. (1967). A monograph on sediment transport in alluvial streams. Teknisk Forlag, Skelbaekgade, Copenhagen V, Denmark, Technical University of Denmark Edition, 64 p.

Hazen, A. (1904). On sedimentation. Transactions of the American Society of Civil Engineers (ASCE), 53(980), pp. 235-244.

Huet, C (1998a). Etude bibliographique pour le choix d'un traceur activable. Note Technique CEA, DAMRI/SAR/S/NT/98-41, 35 p.

Huet, C (1998b). Détermination expérimentale de la limite de détection de différentes terres rares. Note Technique CEA, DAMRI/SAR/S/NT/98-416, 33 p.

Le Guennec, B. (1994). Transport solide et morphologie fluviale. Polycopié de cours. Institut National Polytechnique de Toulouse. Ecole Nâtionale Supérieure d'Electrotechnique, d'Electronique, d'Informatique et d'Hydraulique de Toulouse, $102 \mathrm{p}$.

Neuilly, M. (1993). Modélisation et estimation des erreurs de mesure. Commission d'Etablissement des Méthodes d'Analyses du Commissariat à l'Energie Atomique. Technique et Documentation - Lavoisier, 644 p.

Ramez, P. (1996). Méthodologie de lissage de la géométrie d'un cours d'eau. Approche Globale par Tronçon. Document non publié. Division HydrologieHydraulique. Cemagref Lyon, 9 p.

Renault, D. \& Godaliyadda, G.G.A. (1999). Generic typology for irrigation systems operation. Research Report 29. International Water Management Institute (IWMI), Sri Lanka, 22 p.

Rosgen, D. (1996). Applied river morphology. Edition Widland Hydrology. Pagosa Springs, Colorado, 369 p.

Tola, F. (1982). The use of radioactive tracers in dynamic sedimentology. Part $I$ : Methodology. Part II : Analysis of results. Note Technique CEA-N-2261, $42 \mathrm{p}$.

Vabre, A. \& Le Guennec, B. (1999). Identification of sediment deposition laws on an irrigation canal system in Pakistan. Working Paper WP 2000-03, 15 p. Cemagref Montpellier.

Vabre, A. (1998). Campagne de mesures au moyen de traceurs de juin à novembre.1998 sur Jamrao Canal System. Objectifs et méthode. Rapport CEA, DAMRI/SAR/S/RAP/98-104/V17, 16 p.

Yang, C.T. (1972). Unit stream power and sediment transport. Journal of the Hydraulics Division. Proceedings of the American Society of Civil Engineers (ASCE), Vol. HY 10, pp. 1805-1825. 